\title{
FLOW OF NON-NEWTONIAN FLUID BETWEEN TORSIONALLY OSCILLATING DISCS
}

\author{
By (Miss) G. K. RAJESWARI \\ (Department of Applied Mathematics, Indian Institute of Science, Bangalore-12) \\ Received May 30, 1961
}

(Communicated by Prof. P. L. Bhatnagar, F.A.sc.)

\section{INTRODUCTION}

A THEORETICAL analysis is made of the flow of an incompressible non-Newtonian viscous fluid contained between two torsionally oscillating infinite parallel discs on lines similar to those of the corresponding Newtonian case considered by Rosenblat. We study the two cases where (i) one disc only is oscillating torsionally while the other is at rest and (ii) both the discs oscillate with the same frequency and amplitude but the motions at any instant are in opposite directions. Assuming that the amplitude of the oscillation namely $(\Omega / n)$ is small and neglecting the terms of $0(\Omega / n)^{2}$ in the equations of motion in comparison with terms of $0(\Omega / n)$ we solve exactly for the velocity field and pressure satisfying all the boundary conditions completely. This approximation gives the same transverse velocity field as in the Newtonian case obtained by Rosenblat. We find that the radialaxial flow has a mean steady component and a fluctuating component of frequency twice that of the oscillating disc. We observe that the approximations made gives the velocity field valid only for small Reynolds numbers unlike the Newtonian case, where the asymptotic expansion of the velocity field for large Reynolds numbers has also been obtained. We have studied the non-Newtonian effects on the streamlines of the steady part of the radial-axial flow and on the steady component of the radial velocity in both the cases through a dimensionless parameter $\mathrm{S}=\left(v_{c} / d^{2}\right)$, where $v_{c}$ is the kinematic coefficient of cross-viscosity assumed as constant and $d$ is the distance between the plates. We have compared the flow of the Newtonian case $(\mathrm{S}=0)$ with the non-Newtonian case considering both negative and positive values of $S$. We observe the continuity of the effect as $S$ changes from negative to positive values. We have derived the expressions for the shearing stresses acting on the plates for small Reynolds numbers.

1. We consider a body of non-Newtonian fiuid bounded by two infinite parallel plane discs which are represented by the planes $z=0$ and $z=d$ 188 
in a cylindrical polar co-ordinate system. The dises perform torsional oscillations about the axis, $r=0$. If $w, v, w$ are respectively the radial, transversc and axial velocity components, $p$, the prossure, $\rho$, the density, $\mu, \mu_{c}$, the coefficients of viscosity and cross-viscesity and $\nu, \nu_{c}$, the kinematic coefficients of viscosity, the equations of motion of a non-Newtonian fluid in cylindrical polar co-ordinate system are:

$$
\begin{aligned}
& \frac{\partial u}{\partial t}+u \frac{\partial u}{\partial r}+w \frac{\partial u}{\partial z}-\frac{v^{2}}{r} \\
& =-\frac{1}{\rho} \frac{\partial p}{\partial r}+v\left\{\frac{\partial^{2} u}{\partial r^{2}}+\frac{\partial^{2} u}{\partial z^{2}}+\frac{1}{r} \frac{\partial u}{\partial r}-\frac{u}{r^{2}}\right\} \\
& +2 v_{c}\left\{4 \frac{\partial u}{\partial r} \frac{\partial^{2} u}{\partial r^{2}}+\left(\frac{\partial u}{\partial z}+\frac{\partial w}{\partial r}\right)\left(\frac{\partial^{2} u}{\partial r \partial z}+\frac{\partial^{2} w}{\partial r^{2}}\right)\right. \\
& -\frac{u}{r} \frac{\partial^{2} w}{\partial r \partial z}-\frac{u}{r} \frac{\partial^{2} u}{\partial z^{2}}-\frac{1}{2 r}\left(\frac{\partial u}{\partial z}\right)^{2}+\frac{1}{2 r}\left(\frac{\partial w}{\partial r}\right)^{2} \\
& \left.+\frac{2}{r}\left(\frac{\partial u}{\partial r}\right)^{2}-\frac{2 u^{2}}{r^{3}}\right\}+v_{c}\left\{2\left(\frac{\partial v}{\partial r}-\frac{v}{r}\right) \frac{\partial}{\partial r}\left(\frac{\partial v}{\partial r}-\frac{v}{r}\right)\right. \\
& \left.+\frac{\partial v}{\partial z} \frac{\partial}{\partial z}\left(\frac{\partial v}{\partial r}-\frac{v}{r}\right)-\frac{1}{r}\left(\frac{\partial v}{\partial z}\right)^{2}+\left(\frac{\partial v}{\partial r}-\frac{v}{r}\right) \frac{\partial^{2} v}{\partial z^{2}}\right\}, \\
& \frac{\partial v}{\partial t}+u \frac{\partial v}{\partial r}+w \frac{\partial v}{\partial z}+\frac{u v}{r} \\
& =v\left\{\frac{\partial^{2} v}{\partial r^{2}}+\frac{\partial^{2} v}{\partial z^{2}}+\frac{1}{r} \frac{\partial v}{\partial r}-\frac{v}{r^{2}}\right\}+v_{c}\left\{\frac{\partial^{2} v}{\partial r \partial z}\left(\frac{\partial u}{\partial z}+\frac{\partial w}{\partial r}\right)\right. \\
& +\frac{\partial v}{\partial z} \frac{\partial}{\partial r}\left(\frac{\partial u}{\partial z}+\frac{\partial w}{\partial r}\right)-2 \frac{\partial^{2} u}{\partial r \partial z} \frac{\partial v}{\partial z}+\left(\frac{\partial u}{\partial z}+\frac{\partial w}{\partial r}\right) \frac{\partial}{\partial z} \\
& \times\left(\frac{\partial v}{\partial r}-\frac{v}{r}\right)+\left(\frac{\partial v}{\partial r}-\frac{v}{r}\right) \frac{\partial}{\partial z}\left(\frac{\partial u}{\partial z}+\frac{\partial w}{\partial r}\right) \\
& -2 \frac{\partial w}{\partial z} \frac{\partial}{\partial r}\left(\frac{\partial v}{\partial r}-\frac{v}{r}\right)-2 \frac{\partial^{2} v}{\partial z^{2}} \frac{\partial u}{\partial r}-2 \frac{\partial^{2} w}{\partial r \partial z}\left(\frac{\partial v}{\partial r}-\frac{v}{r}\right) \\
& \left.-\frac{4}{r} \frac{\partial w}{\partial z}\left(\frac{\partial v}{\partial r}-\frac{v}{r}\right)+\frac{2}{r}\left(\frac{\partial u}{\partial z}+\frac{\partial w}{\partial r}\right) \frac{\partial v}{\partial z}\right\},
\end{aligned}
$$




$$
\begin{aligned}
\frac{\partial w}{\partial t}+ & u \frac{\partial w}{\partial r}+w \frac{\partial w}{\partial z} \\
= & -\frac{1}{\rho} \frac{\partial p}{\partial z}+\nu\left\{\frac{\partial^{2} w}{\partial r^{2}}+\frac{\partial^{2} w^{\prime}}{\partial z^{2}}+\frac{1}{r} \frac{\partial w}{\partial r}\right\} \\
& +2 v_{c}\left\{4 \frac{\partial w}{\partial z} \frac{\partial^{2} w}{\partial z^{2}}-\frac{u}{r} \frac{\partial^{2} u}{\partial r} \frac{u}{\partial z}-\frac{\partial^{2} w}{r}-\frac{1}{\partial r^{2}} \frac{\partial u}{\partial r} \frac{\partial u}{\partial z}\right. \\
& \left.-\frac{1}{r} \frac{\partial u}{\partial r} \frac{\partial w}{\partial r}+\left(\frac{\partial u}{\partial z}+\frac{\partial w}{\partial r}\right)\left(\frac{\partial^{2} u}{\partial z^{2}}+\frac{\partial^{2} w}{\partial r \partial z}\right)\right\} \\
& +v_{c}\left\{\frac{\partial v}{\partial z}\left(2 \frac{\partial^{2} v}{\partial z^{2}}+\frac{\partial^{2} v}{\partial r^{2}}\right)+\frac{\partial^{2} v}{\partial r \partial z}\left(\frac{\partial v}{\partial r}-\frac{v}{r}\right)\right\},
\end{aligned}
$$

and

$$
\frac{\partial u}{\partial r}+\frac{u}{r}+\frac{\partial w}{\partial z}=0
$$

\section{One Disc Oscillating}

We consider the disc at $z=0$ to perform torsional oscillations of frequency $n$ and angular speed $\Omega$, while the disc at $z=d$, remains at rest.

Following Rosenblat, we assume the velocity field as

$$
\left.\begin{array}{l}
u=r \frac{\Omega^{2}}{n} \frac{\partial}{\partial y} \mathrm{~F}(y, \tau), \\
v=r \Omega e^{i \tau} g(y), \\
w=-2 d \frac{\Omega^{2}}{n} \mathrm{~F}(y, \tau),
\end{array}\right\}
$$

where

$$
y=\frac{z}{d} \quad \text { and } \quad \tau=n t \text {. }
$$

Further we assume that

$$
\underset{\rho}{p}=\frac{\Omega^{2} r^{2}}{2} \mathrm{P}(y, \tau)+2 \Omega^{2} d^{2} \mathrm{~K}(y, \tau) .
$$

The equation of continuity (1.4) is identically satisfied by such a choice of velocity field. The boundary conditions of the problem are

$$
u=w=0, \quad v=\operatorname{Re}\left(r \Omega e^{i n t}\right) \quad \text { at } z=0,
$$


Flow of Non-Newtonian Fluid between Torsionally Oscillating Discs 191

and

$$
u=v=w=0 \quad \text { at } \quad z=d .
$$

Sibstituting (2.1) and (2.2) in the equations of motion (1.1) to (1.3) we have

$$
\begin{aligned}
\frac{\partial^{2} \mathrm{~F}}{\partial y \partial \tau}+ & \left(\frac{\Omega}{n}\right)^{2}\left[\left(\frac{\partial \mathrm{F}}{\partial y}\right)^{2}-2 \mathrm{~F} \frac{\partial^{2} \mathrm{~F}}{\partial y^{2}}\right]-\left[e^{i r} g(y)\right]^{2} \\
= & -\mathrm{P}(y, \tau)+\frac{1}{\mathrm{R}} \frac{\partial^{3} \mathrm{~F}}{\partial y^{3}}+\mathrm{S}\left(\frac{\Omega}{n}\right)^{2}\left[\left(\frac{\partial^{2} \mathrm{~F}}{\partial y^{2}}\right)^{2}-\frac{\partial \mathrm{F}}{\partial y} \frac{\partial^{3} \mathrm{~F}}{\partial y^{3}}\right] \\
& -\mathrm{S}\left[e^{i \tau} \frac{d g}{d y}\right]^{2} \\
\operatorname{ig}(y)+ & 2\left(\frac{\Omega}{n}\right)^{2}\left[g \frac{\partial \mathrm{F}}{\partial y}-\mathrm{F} \frac{d g}{d y}\right] \\
= & \frac{1}{\mathrm{R}} \frac{d^{2} g}{d y^{2}}+2\left(\frac{\Omega}{n}\right)^{2} \mathrm{~S}\left[\frac{d g}{d y} \frac{\partial^{2} \mathrm{~F}}{\partial y^{2}}-\frac{\partial \mathrm{F}}{\partial y} \frac{\partial^{2} g}{d y^{2}}\right],
\end{aligned}
$$

and

$$
\begin{aligned}
2 \frac{\partial \mathrm{F}}{\partial \tau}- & 4\left(\frac{\Omega}{n}\right)^{2} \mathrm{~F} \frac{\partial \mathrm{F}}{\partial y} \\
= & \frac{r^{2}}{d^{2}}\left[\frac{1}{2} \frac{\partial \mathrm{P}}{\partial y}-2 \mathrm{~S} e^{2 i r} \frac{d g}{d y} \frac{d^{2} g}{d y^{2}}-2 \mathrm{~S}\left(\frac{\Omega}{n}\right)^{2} \frac{\partial^{2} \mathrm{~F}}{\partial y^{2}} \frac{\partial^{3} \mathrm{~F}}{\partial y^{3}}\right] \\
& \quad+\frac{2}{\mathrm{R}} \frac{\partial^{2} \mathrm{~F}}{\partial y^{2}}+2 \frac{\partial \mathrm{K}}{\partial y}-28 \mathrm{~S}\left(\frac{\Omega}{n}\right)^{2} \frac{\partial \mathrm{F}}{\partial y} \frac{\partial^{2} \mathrm{~F}}{\partial y^{2}}
\end{aligned}
$$

where

$$
\mathrm{S}=\frac{\nu_{c}}{d^{2}} \quad \text { and } \quad \mathrm{R}=\frac{n d^{2}}{v}
$$

The boundary conditions transform to

$$
\mathrm{F}=\frac{\partial \mathrm{F}}{\partial y}=0, \quad g=1 \quad \text { at } y=0,
$$

and

$$
\mathrm{F}=\frac{\partial \mathrm{F}}{\partial y}=g=0 \quad \text { at } y=1
$$


On the assumption that the amplitude of the oscillations namely $\Omega / n$ is s nall, retaining only the first order terms in $\Omega / n$, we have for the traniverse velocity component

$$
i g(y)=\frac{1}{\mathbf{R}} \frac{d^{2} g}{d y^{2}}
$$

with

$$
g(0)=1 \quad \text { and } \quad g(1)=0 . \quad\}
$$

The solution of $(2.10)$ is

$$
g(y)=\frac{\sinh \sqrt{ } \tilde{i} \mathrm{R}(1-y)}{\sinh \sqrt{ } i \overline{\mathrm{R}}},
$$

same as obtained by Rosenblat in the Newtonian case.

For small Reynolds numbers we have

$$
\begin{aligned}
\frac{v}{r \Omega}= & (1-y)\left\{\left[1-\frac{\mathrm{R}^{2}}{360} y\left(8+8 y-12 y^{2}+3 y^{3}\right)\right] \cos n t\right. \\
& \left.+\frac{\mathrm{R}}{6} y(2-y) \sin n t\right\}+0\left(\mathrm{R}^{3}\right) .
\end{aligned}
$$

Naglecting the terms of oider $(\Omega / n)^{2}$, we have from (2.5) for the radial-axial component of velocity

$$
\frac{\partial^{2} \mathrm{~F}}{\partial y \partial \tau}-\left[g e^{i \tau}\right]^{2}=-\mathrm{P}(y, \tau)+\frac{1}{\mathrm{R}} \frac{\partial^{3} \mathrm{~F}}{\partial y^{3}}-\mathrm{S}\left[e^{i \tau} \frac{d g}{d y}\right]^{2} .
$$

From (2.7) we have

$$
\frac{\partial \mathrm{F}}{\partial \tau}=\frac{1}{\mathrm{R}} \frac{\partial^{2} \mathrm{~F}}{\partial y^{2}}+\frac{\partial \mathrm{K}}{\partial y}
$$

and equating the coefficient of $r^{2}$ to zero we have

$$
\frac{1}{2} \frac{\partial \mathrm{P}}{\partial y}-2 \mathrm{~S} e^{2 i r} \frac{d g}{d y} \frac{d^{2} g}{d y^{2}}=0
$$

which determines $\mathrm{P}(y, r)$.

Let

$$
\mathrm{F}(y, \tau)=f(y)+h(y) e^{2 i \tau},
$$


and

$$
\mathrm{K}(y, \tau)=\mathrm{K}_{0}(y)+\mathrm{K}_{1}(y) e^{2 i \tau} .
$$

From (2.14) we have

$$
\frac{\mathrm{P}}{2}-\mathrm{S} e^{2 i \tau}\left(\frac{d g}{d y}\right)^{2}=a(\tau) .
$$

Substituting (2.15), (2.16) and (2.17) in (2.12) and (2.13) we have

$$
\begin{aligned}
& 2 i h^{\prime}(y) e^{2 i \tau}-\left[g e^{i \tau}\right]^{2} \\
& \quad=-2 a(\tau)-3 \mathrm{~S}\left[e^{i \tau} \frac{d g}{d y}\right]^{2}+\frac{1}{\mathrm{R}}\left[f^{\prime \prime \prime}(y)+h^{\prime \prime \prime}(y) e^{2 i \tau}\right],
\end{aligned}
$$

and

$$
\begin{aligned}
2 i h(y) e^{2 i \tau}=\frac{1}{\mathrm{R}}\left[f^{\prime \prime \prime}(y)+h^{\prime \prime \prime}(y) e^{2 i \tau}\right]+\frac{d}{d y} \mathbf{K}_{\mathbf{0}}(y) \\
+\frac{d}{d y} \mathrm{~K}_{\mathbf{1}}(y) e^{2 i \tau}
\end{aligned}
$$

These equations are sufficient to determine the unknowns $f(y), h(y), \mathrm{K}_{\mathbf{0}}(y)$ and $\mathrm{K}_{1}(y)$. The above equations indicate that the radial-axial flow has a mean steady component and a fluctuating component of frequency twice that of the oscillating plate. From (2.11) we have

$$
[g(y)]^{2} e^{2 i r}=\frac{1}{2}\left[\frac{\cosh \lambda y_{1}-\cos \lambda y_{1}}{\cosh \lambda-\cos \lambda}\right]+\frac{1}{2}\left[\frac{\cosh \lambda(1+i) y_{1}-1}{\cosh \lambda(1+i)-1}\right] e^{2 i \tau},
$$

and

$$
\begin{aligned}
{\left[\frac{d g}{d y}\right]^{2} e^{2 i \tau}=\frac{\lambda^{2}}{4} } & {\left[\frac{\cosh \lambda y_{1}+\cos \lambda y_{1}}{\cosh \lambda-\cos \lambda}\right] } \\
& +\frac{i \lambda^{2}}{4}\left[\frac{\cosh \lambda(1+i) y_{1}+1}{\cosh \lambda(1+i)-1}\right] e^{2 i \tau},
\end{aligned}
$$

where

$$
\lambda=\sqrt{2 \mathrm{R}} \quad \text { and } \quad y_{1}=1-y .
$$


Substitution of (2.20) and (2.21) into (2.18) yields

$$
\frac{f^{\prime \prime \prime}(y)}{\mathrm{R}}=L_{0}+3 \mathrm{~S} \frac{\lambda^{2}}{4}\left[\frac{\cosh \lambda y_{1}+\cos \lambda y_{1}}{\cosh \lambda-\cos \lambda}\right]-\frac{1}{2}\left[\frac{\cosh \lambda y_{1}-\cos \lambda y_{1}}{\cosh \lambda-\cos \lambda}\right],
$$

and

$$
\begin{aligned}
\frac{1}{\mathrm{R}} h^{\prime \prime \prime}(y) & -2 i h^{\prime}(y) \\
= & \mathrm{L}_{1}+3 \mathrm{~S} \frac{\lambda^{2}}{4}\left[\frac{\cosh \lambda(1+i) y_{1}+1}{\cosh \lambda(1+i)-1}\right] \\
& -\frac{1}{2}\left[\frac{\cosh \lambda(1+i) y_{1}-1}{\cosh \lambda(1+i)-1}\right]
\end{aligned}
$$

where we have taken

$$
2 \alpha(\tau)=\mathrm{L}_{0}+\mathrm{L}_{1} e^{2 i \tau} \text {. }
$$

The boundary conditions $(2.8),(2.9)$ reduce to

$$
f=f^{\prime}=0 \quad \text { at } y=0 \quad \text { and } \quad y=1 \text {, }
$$

and

$$
h=h^{\prime}=0 \quad \text { at } y=0 \quad \text { and } \quad y=1 .
$$

The solution of (2.22) for the mean steady component under the conditions $(2.24)$ is found to be

$$
\begin{aligned}
& f(y)=\left[\frac{1}{\cosh \lambda-\cos \lambda}\right]\left\{\left[\frac{1}{4 \lambda}\left(\sinh \lambda y_{1}+\sin \lambda y_{1}\right)\right.\right. \\
&+\frac{1}{4} y(1-y)^{2}(\cosh \lambda+\cos \lambda)-\frac{1}{4 \lambda}\left(1-3 y^{2}+2 y^{3}\right) \\
&\left.\times(\sinh \lambda+\sin \lambda)-\frac{1}{2} y^{2}(1-y)\right]+3 S \frac{\lambda}{8} \\
&\left.\times\left[\left(2 y^{3}-3 y^{2}+1\right)(\sinh \lambda-\sin \lambda)-\left(\sinh \lambda y_{1}-\sin \lambda y_{1}\right)\right]\right\} \\
&-3 S \frac{\lambda^{2}}{8} y(1-y)^{2},
\end{aligned}
$$


and

$$
\begin{aligned}
& f^{\prime}(y)=\left[\frac{1}{\cosh \lambda-\cos \lambda}\right]\left\{\left[-\frac{1}{4}\left(\cosh \lambda y_{1}+\cos \lambda y_{1}\right)\right.\right. \\
&+\frac{3}{2 \lambda} y(1-y)(\sinh \lambda+\sin \lambda)+\frac{1}{4}\left(1-4 y+3 y^{2}\right) \\
&\left.\times(\cosh \lambda+\cos \lambda)-\frac{1}{2} y(2-3 y)\right] \\
&+3 S \frac{\lambda^{2}}{8}\left[\cosh \lambda y_{1}-\cos \lambda y_{1}\right)+\frac{6}{\lambda} y(y-1) \\
&\times(\sinh \lambda-\sin \lambda)]\}+3 S \frac{\lambda^{2}}{8}\left(4 y-1-3 y^{2}\right)
\end{aligned}
$$

For small Reynolds numbers we have

$$
\begin{aligned}
f(y) \approx & \frac{\mathrm{R}}{120} y^{2}(1-y)^{2}\left\{(3-y)+\frac{\mathbf{R}^{2}}{3780}\left[-5 y^{5}+35 y^{4}\right.\right. \\
& \left.-105 y^{3}+175 y^{2}-133 y-21\right] \\
& \left.\left.+\frac{\mathbf{R}^{2} S}{7}\left[y^{3}-5 y^{2}+10 y-10\right)\right]\right\}+0\left(\mathbf{R}^{5}\right),
\end{aligned}
$$

and

$$
\begin{aligned}
f^{\prime}(y) \approx & \frac{\mathrm{R}}{120} y(1-y)\left\{\left(6-15 y+5 y^{2}\right)\right. \\
& -\frac{\mathrm{R}^{2}}{3780}\left[42-1365 y^{2}+45\left(-y^{6}+7 y^{5}-21 y^{4}+35 y^{3}+7 y\right)\right] \\
& \left.\quad-\frac{\mathrm{R}^{2} \mathrm{~S}}{7}\left[7\left(y^{4}-5 y^{3}+10 y^{2}-10 y\right)+20\right]\right\}+0\left(\mathrm{R}^{5}\right) .
\end{aligned}
$$

The solutions for $\mathbf{S}=0$, corresponding to the Newtonian case are in agreement with those of Rosenblat.

The unsteady component of the radial-axial flow is given by the equation (2.23). We have

$$
\begin{gathered}
4[\cosh \lambda(1+i)-1]\left[\cosh \frac{\lambda(1+i)}{\sqrt{ } 2}-1-\frac{\lambda(1+i)}{2 \sqrt{ } 2}\right. \\
\left.\sinh \frac{\lambda(1+i)}{\sqrt{2}}\right] h(y)
\end{gathered}
$$




$$
\begin{aligned}
= & \left.3 \mathrm{~S} \frac{\lambda^{2}}{4}+\frac{i}{2}\right)\left\{\left[1-\cosh \lambda \frac{(1+i)}{\sqrt{ } 2}\right][y(1+\cosh \lambda(1+i))\right. \\
& +\frac{\sqrt{ } 2}{\lambda(1+i)} \sinh \frac{\lambda(1+i)}{\sqrt{ } 2} y(\cosh \lambda(1+i)-1) \\
& -\frac{2}{\lambda(1+i)} \sinh \lambda(1+i) \cosh \frac{\lambda(1+i)}{\sqrt{ } 2} y \\
& \left.+\frac{2}{\lambda(1+i)} \sinh \lambda(1+i) y_{1}\right]+\left[1-\cosh \frac{\lambda(1+i)}{\sqrt{ } 2} y\right] \\
& \times\left[\left(-1+\cosh \lambda(1+i) \cosh \frac{\lambda(1+i)}{\sqrt{ } 2}-\frac{1}{\sqrt{ } 2}\right.\right. \\
& \times \sinh \lambda(1+i) \sinh \frac{\lambda(1+i)}{\sqrt{ } 2}+\frac{\sinh \lambda(1+i)}{\lambda(1+i)} \\
& \times \cosh \frac{\lambda(1+i)}{\sqrt{ } 2}+\frac{\sqrt{ } 2}{\lambda(1+i)} \sinh \frac{\lambda(1+i)}{\sqrt{ } 2} \\
& \left.\times(1-\cosh \lambda(1+i))-\frac{\sinh \lambda(1+i)}{\lambda(1+i)}\right] \\
& +\left[\frac { 1 } { \sqrt { } 2 } \operatorname { s i n h } \lambda ( 1 + i ) \operatorname { s i n h } \frac { \lambda ( 1 + i ) } { \sqrt { } 2 } \left\{y-\frac{\sqrt{ } 2}{\lambda(1+i)}\right.\right. \\
& \left.\times \sinh \frac{\lambda(1+i)}{\sqrt{ } 2} y\right\}-\frac{1}{\sqrt{ } 2} \sinh \frac{\lambda(1+i)}{\sqrt{ } 2} \\
& \times\left\{-\sinh \lambda(1+i) y_{1}+\sinh \lambda(1+i) \cosh \frac{\lambda(1+i)}{\sqrt{ } 2} y\right. \\
&
\end{aligned}
$$

For small Reynolds numbers we get,

$$
\begin{aligned}
h(y) \approx \frac{\mathrm{R}}{120} y^{2}(1-y)^{2}\{(3-y)(1-3 i \mathrm{RS}) \\
\left.-\frac{i \mathrm{R}}{210}\left(30 y^{3}-150 y^{2}+167 y+29\right)\right\}+0\left(\mathbf{R}^{3}\right) .
\end{aligned}
$$


The shearing stresses for the velocity field (2.1) are given by

$$
\begin{aligned}
& \mathrm{T}_{r z}=\frac{\partial u}{\partial z}\left[\left(\mu-2 \mu_{\mathrm{c}} \frac{u}{r}\right],\right. \\
& \mathrm{T}_{\theta z}=\frac{\partial v}{\partial z}\left[\mu-2 \mu_{\mathrm{c}} \frac{\partial u}{\partial r}\right],
\end{aligned}
$$

and

$$
\mathrm{T}_{r_{\theta}}=\mu_{c} \frac{\partial u}{\partial z} \frac{\partial v}{\partial z}
$$

For small Reynolds numbers the shearing stresses acting on the plate $z=0$ are given by

$$
\begin{aligned}
& \mathrm{T}_{r z}=\mu \frac{r}{d} \frac{\Omega^{2}}{n} \frac{\mathrm{R}}{20}\left[1+\cos 2 n t+\mathrm{R}\left(3 \mathrm{~S}+\frac{29}{630}\right) \sin 2 n t\right]+0\left(\mathrm{R}^{3}\right), \\
& \mathrm{T}_{\theta z}=-\mu \frac{r}{d} \Omega\left[\left(1+\frac{\mathbf{R}^{2}}{45}\right) \cos n t-\frac{\mathrm{R}}{3} \sin n t\right]+0\left(\mathrm{R}^{3}\right),
\end{aligned}
$$

and

$$
\begin{aligned}
\mathrm{T}_{\tau \theta}=- & \mu_{c} \frac{r^{2}}{d^{2}} \frac{\Omega^{3}}{n} \frac{\mathrm{R}}{40}[\cos 3 n t+3 \cos n t \\
& \left.+\mathrm{R}\left(3 \mathrm{~S}-\frac{181}{630}\right)(\sin 3 n t+\sin n t)\right]+0\left(\mathrm{R}^{3}\right) .
\end{aligned}
$$

The shearing stresses acting on the disc $z=d$ are

$$
\begin{aligned}
& \mathrm{T}_{r z}=\mu \frac{r}{d} \frac{\Omega^{2}}{n} \frac{\mathrm{R}}{30}\left[1+\cos 2 n t+\mathrm{R}\left(3 \mathrm{~S}+\frac{19}{105}\right) \sin 2 n t\right]+0\left(\mathrm{R}^{3}\right) . \\
& \mathrm{T}_{\theta z}=-\mu \frac{r}{d} \Omega\left[\left(1-\frac{7 \mathrm{R}^{2}}{360}\right) \cos n t+\frac{\mathrm{R}}{6} \sin n t\right]+0\left(\mathrm{R}^{3}\right),
\end{aligned}
$$

and

$$
\begin{aligned}
\mathrm{T}_{r \theta}= & -\mu_{\mathbf{c}} \frac{r^{2}}{d^{2}} \frac{\Omega^{3}}{n} \frac{\mathrm{R}}{60}[\cos 3 n t+3 \cos n t \\
& \left.+\mathrm{R}\left(3 \mathrm{~S}+\frac{73}{210}\right)(\sin 3 n t+\sin n t)\right]+0\left(\mathrm{R}^{\mathbf{3}}\right) .
\end{aligned}
$$


Figure 1 represents the effect of non-Newtonian term on the typical streamlines of the steady component of the flow. We have drawn the curves for $S=-0 \cdot 5,-0 \cdot 1,0$ and $+0 \cdot 05$. We observe the continuity of the effect as the parameter ' $S$ ' changes from negative to positive values. The negative non-Newtonian coefficient flattens the strcamline while the positive coefficient makes it more curved. Figure 2 shows the dimensionless steady

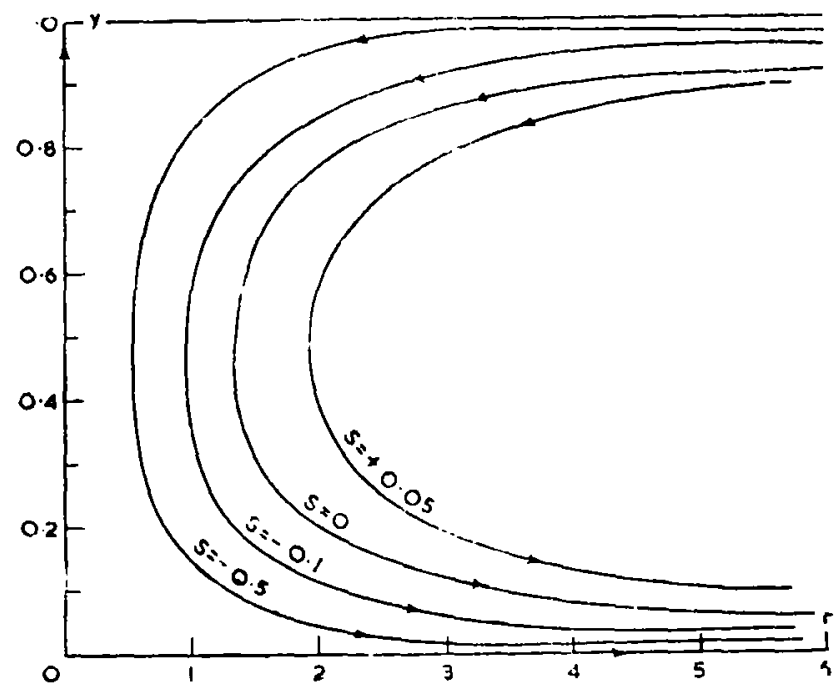

FIG. 1. One disc oscillating: Streamlines of steady radial-axisi f.c $и$ fcr $R=5$ and $S=-0.5-0.1,0,+0.05$.

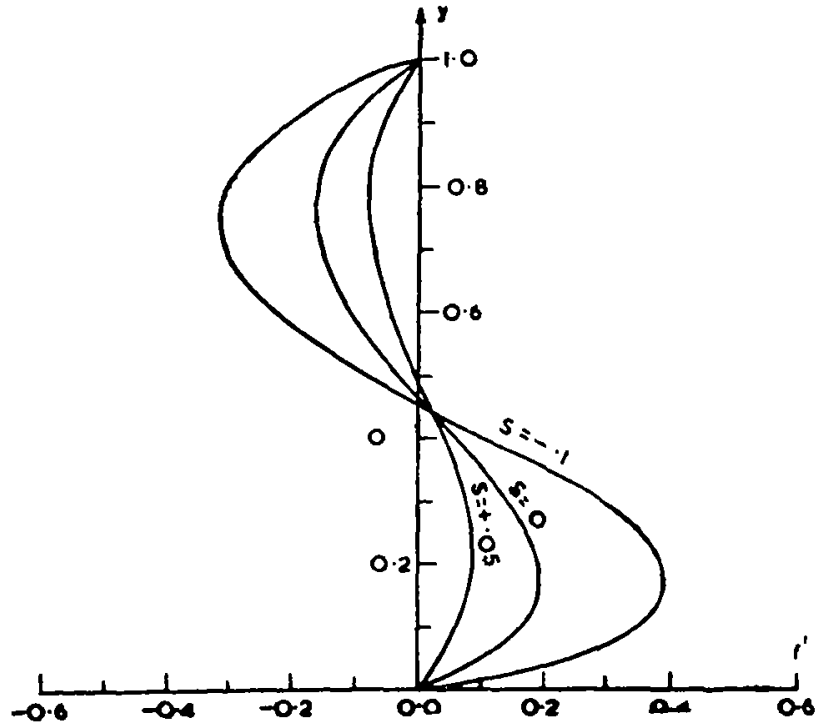

Fio. 2. One disc oscillating: Steady radial velocity $f^{\prime}$ for $\mathrm{S}=-0.1,0 .+0.05$. 
component of the radial velocity, namely, $f^{\prime}$ for $S=-0 \cdot 1,0$ and +0.05 . We find $f^{\prime}=0$ at $y=0.45$ for $\mathrm{S}=-0.1$ and at $y=0.48$ for $\mathrm{S}=+0.05$.

\section{Both Discs Oscillating}

We now consider the case where the two discs are oscillating with same frequency and angular speed but in opposite directions. The boundary conditions in the present case are

$$
u=w=0, \quad v=\operatorname{Re}\left(r \Omega e^{i n t}\right) \quad \text { at } z=0,
$$

and

$$
\left.u=w=0, \quad v=-\operatorname{Re}\left(r \Omega e^{i n t}\right) \quad \text { at } z=d . \quad\right\}
$$

The equations of motion (1.1) to (1.3) can again be transformed by the choice of the velocity field (2.1) in a similar way and for the transverse flow we have

$$
\frac{d^{2} g}{\bar{d} y^{2}}-i \mathrm{Rg}=0
$$

as previously but with $g(0)=1, g(1)=-1$.

The solution now is

$$
g(y)=\frac{\sinh \sqrt{i \mathrm{R}} y_{1}-\sinh \sqrt{i \overline{\mathrm{R}} y}}{\sinh \sqrt{ } i \overline{\mathrm{R}}},
$$

once again the same as that obtained by Rosenblat.

For small Reynolds numbers this yields the velocity profile

$$
\begin{gathered}
\frac{v}{r \Omega} \approx(1-2 y)\left\{\left[1-\frac{\mathrm{R}^{2}}{360} v(1-y)\left(1+3 y-3 y^{2}\right)\right] \cos n t\right. \\
\left.+\frac{\mathrm{R}}{6} y(1-y) \sin r t\right\}+0\left(\mathrm{R}^{3}\right) .
\end{gathered}
$$

From (3.3) we have

$$
\begin{aligned}
{[g(y)]^{2} e^{2 i \tau}=} & \frac{\cosh \frac{\lambda}{2}+\cos \frac{\lambda}{2}}{\cosh \lambda-\cos \lambda}\left[\cosh \frac{\lambda}{2}(1-2 y)-\cos \frac{\lambda}{2}(1-2 y)\right] \\
& \left.+\frac{\cosh \frac{\lambda}{2}(1+i)-1}{\cosh \lambda(1+i)-1}\left[\cosh \frac{\lambda}{2}(1+i)(1-2 y)-1\right)\right] e^{2 i \tau},
\end{aligned}
$$


and

$$
\begin{aligned}
{\left[\frac{d g}{d y}\right]^{2} e^{2 i \tau}=} & \frac{\lambda^{2}}{2} \frac{\cosh \frac{\lambda}{2}+\cos \frac{\lambda}{2}}{\cosh \lambda-\cos \lambda}\left[\cosh \frac{\lambda}{2}(1-2 y)+\cos \frac{\lambda}{2}(1-2 \mathrm{y})\right] \\
& +\frac{i \lambda^{2}}{2} \frac{\cosh \frac{\lambda}{2}(1+i)+1}{\cosh \lambda(1+i)-1} \\
& \times\left[\cosh \frac{\lambda}{2}(1+i)(1-2 y)+1\right] e^{2 i \tau}
\end{aligned}
$$

Substitution of (3.5) and (3.6) in equation (2.18) yields the cquations necessary for determining the steady and the fluctuating componcnts of the radial-axial flow under the same boundary conditions (2.24) and (2.25). The steady components of the axial and radial flows are

$$
\begin{aligned}
f(y)=- & \frac{1}{2} \frac{\cosh \frac{\lambda}{2}+\cos \frac{\lambda}{2}}{\cosh \lambda-\cos \lambda}\left\{( 3 \mathrm { S } \frac { \lambda ^ { 2 } } { 2 } - 1 ) \left[\left(3 y^{2}-2 y^{3}\right)\right.\right. \\
& \times\left(\frac{2}{\lambda} \sinh \frac{\lambda}{2}-\cosh \frac{\lambda}{2}\right)+\frac{1}{\lambda} \sinh \frac{\lambda}{2}(1-2 y)+y \cosh \frac{\lambda}{2} \\
& \left.-\frac{1}{\lambda} \sinh \frac{\lambda}{2}\right]+\left(3 \mathrm{~S} \frac{\lambda^{2}}{2}+1\right)\left[\left(3 y^{2}-2 y^{3}\right)\left(\cos \frac{\lambda}{2}-\frac{2}{\lambda} \sin \frac{\lambda}{2}\right)\right. \\
& \left.\left.-\frac{1}{\lambda} \sin \frac{\lambda}{2}(1-2 y)-y \cos \frac{\lambda}{2}+\frac{1}{\lambda} \sin \frac{\lambda}{2}\right]\right\},
\end{aligned}
$$

and

$$
\begin{aligned}
f^{\prime}(y)= & \frac{1}{2} \frac{\cosh \frac{\lambda}{2}+\cos \frac{\lambda}{2}}{\cosh \lambda-\cos \lambda}\left\{( 3 \mathrm { S } \frac { \lambda ^ { 2 } } { 2 } - 1 ) \left[6 ( y ^ { 2 } - y ) \left(\frac{2}{\lambda} \sinh \frac{\lambda}{2}\right.\right.\right. \\
& \left.\left.-\cosh \frac{\lambda}{2}\right)-\cosh \frac{\lambda}{2}+\cosh \frac{\lambda}{2}(1-2 y)\right]+\left(3 \mathrm{~S} \frac{\lambda^{2}}{2}+1\right) \\
& \times\left[6\left(y^{2}-y\right)\left(\cos \frac{\lambda}{2}-\frac{2}{\lambda} \sin \frac{\lambda}{2}\right)+\cos \frac{\lambda}{2}\right. \\
& \left.\left.-\cos \frac{\lambda}{2}(1-2 y)\right]\right\}
\end{aligned}
$$


For small Reynolds numbers we have

$$
\begin{aligned}
f(y) \approx & \frac{\mathbf{R}}{60} y^{2}(1-y)^{2}(1-2 y)\left\{-1+\frac{\mathbf{R}^{2}}{3 \times 7 !}[-3+20 y\right. \\
& \left.\left.\left(y^{3}-2 y^{2}+2 y-1\right)\right]-\frac{\mathbf{R}^{2} \mathrm{~S}}{28}\left[4 y^{2}-4 y+3\right]\right\}+0\left(\mathbf{R}^{5}\right),
\end{aligned}
$$

and

$$
\begin{aligned}
f^{\prime}(y) \approx & \frac{\mathrm{R}}{30} y(1-y)\left\{\left(1-5 y+5 y^{2}\right)+\frac{\mathrm{R}^{2}}{7 !}\left[60\left(y^{6}-3 y^{5}+4 y^{4}-3 y^{3}\right)\right.\right. \\
& +5 y(13 y-1)-1]-\frac{\mathrm{R}^{2} \mathrm{~S}}{28}\left[7 \left(4 y^{4}-8 y^{3}\right.\right. \\
& \left.\left.\left.+7 y^{2}-3 y\right)+3\right]\right\}+0\left(\mathrm{R}^{5}\right) .
\end{aligned}
$$

The fluctuating component of the radial-axial flow is given by

$$
\begin{aligned}
\frac{\lambda(1+i)}{\sqrt{ } 2} & h(y) \\
=\frac{i \mathrm{~L}_{1}}{2} & {\left[\frac{\lambda(1+i)}{\sqrt{ } 2} y-\sinh \frac{\lambda(1+i)}{\sqrt{ }} y\right]+\mathrm{D} \frac{\lambda(1+i)}{\sqrt{ } 2} } \\
& \times\left[1-\cosh \frac{\lambda(1+i)}{\sqrt{ } 2} y\right]+\frac{3 \mathrm{~S} \frac{\lambda^{2}}{4}+\frac{i}{2}}{\cosh \lambda(1+i)-1} \\
& \times\left\{-\frac{\lambda(1+i)}{\sqrt{ } 2} y+\frac{1}{\sqrt{ } 2} \sinh \lambda(1+i) \cosh \frac{\lambda}{2}(1+i)\right. \\
& \times \cosh \frac{\lambda(1+i)}{\sqrt{ } 2} y-\sinh \frac{\lambda(1+i)}{\sqrt{ } 2} \sinh \frac{\lambda(1+i)}{\sqrt{ } 2} y \\
& \left.-\frac{1}{\sqrt{ } 2} \cosh \frac{\lambda}{\sqrt{ } 2}(1+i) \sinh \frac{\lambda}{2}(1+i)(1-2 y)\right\} \\
+ & \frac{\left(3 \mathrm{~S} \frac{\lambda^{2}}{4}-\frac{i}{2}\right)}{\cosh \lambda(1+i)-1}\left\{\frac{1}{\sqrt{ } 2} \sinh \frac{\lambda}{2}(1+i) \cosh \frac{\lambda(1+i)}{\sqrt{ } 2} y\right. \\
& \left.-\frac{\lambda(1+i)}{\sqrt{ } 2} y \cosh \frac{\lambda(1+i)}{2}-\frac{1}{\sqrt{ } 2} \sinh \frac{\lambda}{2}(1+i)(1-2 y)\right\},
\end{aligned}
$$


where

$$
\begin{aligned}
\frac{i \mathrm{~L}_{1}}{2}[\cosh & \lambda(1+i)-1]\left[2 \cosh \frac{\lambda(1+i)}{\sqrt{ } 2}\right. \\
& \left.-2-\frac{\lambda(1+i)}{\sqrt{ } 2} \sinh \frac{\lambda(1+i)}{\sqrt{ } 2}\right] \\
= & \left.3 \mathrm{~S} \frac{\lambda^{2}}{4}-\frac{i}{2}\right)\left\{\sqrt{ } 2 \sinh \frac{\lambda}{2}(1+i) \sinh \frac{\lambda}{\sqrt{ } 2}(1+i)\right. \\
& \left.-\frac{\lambda(1+i)}{\sqrt{ } 2} \cosh \frac{\lambda}{2}(1+i) \sinh \frac{\lambda}{\sqrt{ } 2}(1+i)\right\} \\
& +\left(3 \mathrm{~S} \frac{\lambda^{2}}{4}+\frac{i}{2}\right)\left\{\sqrt{ } 2 \sinh \frac{\lambda}{2}(1+i) \cosh \frac{\lambda(1+i)}{\sqrt{ } 2}\right. \\
& \times \sinh \frac{\lambda}{\sqrt{ } 2}(1+i)+2 \sinh ^{2} \frac{\lambda(1+i)}{2}\left(1-\cosh \frac{\lambda(1+i)}{\sqrt{2}}\right) \\
& \left.-\frac{\lambda(1+i)}{\sqrt{ } 2} \sinh \frac{\lambda}{\sqrt{ } 2}(1+i)\right\},
\end{aligned}
$$

and

$$
\begin{aligned}
& \mathrm{D}[\cosh \lambda(1+i)-1]\left[2 \cosh \frac{\lambda(1+i)}{\sqrt{ } 2}\right. \\
& \left.-2-\frac{\lambda(1-i)}{\sqrt{ } 2} \sinh \frac{\lambda(1+i)}{\sqrt{ } 2}\right] \\
& =-\left(3 \mathrm{~S} \frac{\lambda^{2}}{4}-\frac{i}{2}\right)\left\{\cosh \frac{\lambda}{2}(1+i)\left(1-\cosh \frac{\lambda(1+i)}{\sqrt{2}}\right)\right. \\
& \left.+\frac{1}{\sqrt{ } 2} \sinh \frac{\lambda}{2}(1+i) \sinh \frac{\lambda}{\sqrt{ } 2}(1+i)\right\}-\left(3 S \frac{\lambda^{2}}{4}+\frac{i}{2}\right) \\
& \times\left\{\cosh ^{2} \frac{\lambda}{2}(1+i)\left(1-\cosh \frac{\lambda(1+i)}{\sqrt{ } 2}\right)\right. \\
& \left.+\frac{1}{\sqrt{ } / 2} \sinh \frac{\lambda}{2}(1+i) \cosh \frac{\lambda}{2}(1-i) \sinh \frac{\lambda}{\sqrt{ } 2}(1+i)\right\} \text {. }
\end{aligned}
$$

In the present investigation we find that our approximations are valid for small Reynolds numbers only. Figure 3 shows the effect of non-Newtonian term on the typical streamline of the steady flow when both the discs are oscillating in the opposite directions. The flow is symmetrical about 
the plane $y=\frac{1}{2}$. Curves are drawn for $R=10$ and $S=-0 \cdot 5,-0 \cdot 1,0$, +0.05 . The effect of negative non-Newtonian coefficient is to flatten the streamline and that of the positive non-Newtonian coefficient is to make it more curved. Figure 4 represents the steady component of the radial flow

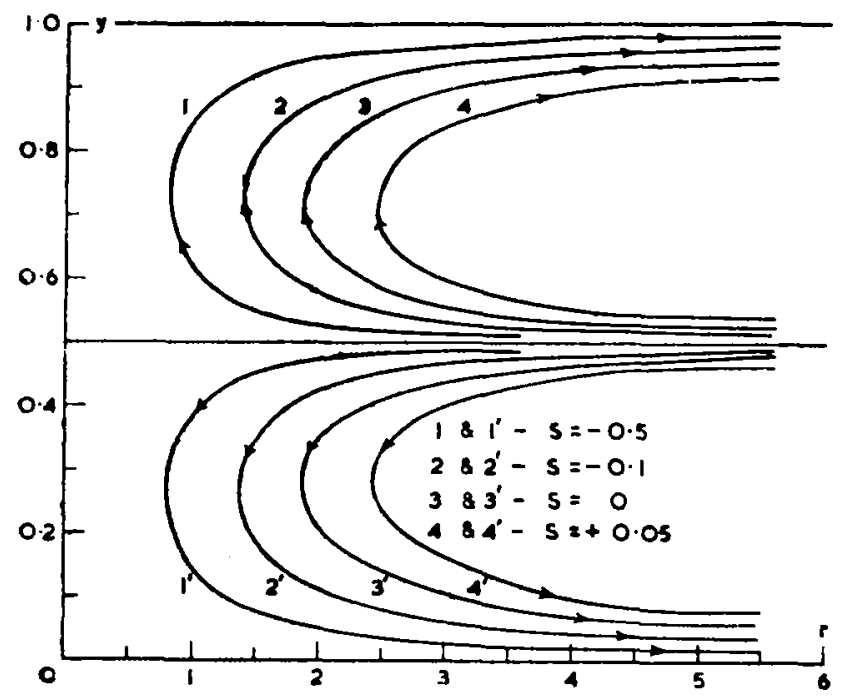

Fro. 3. Two discs oscillating: Streamlines of steady radial-axial flow for $R=10$ and $S=-0.5 .-0.1,0,+0.05$.

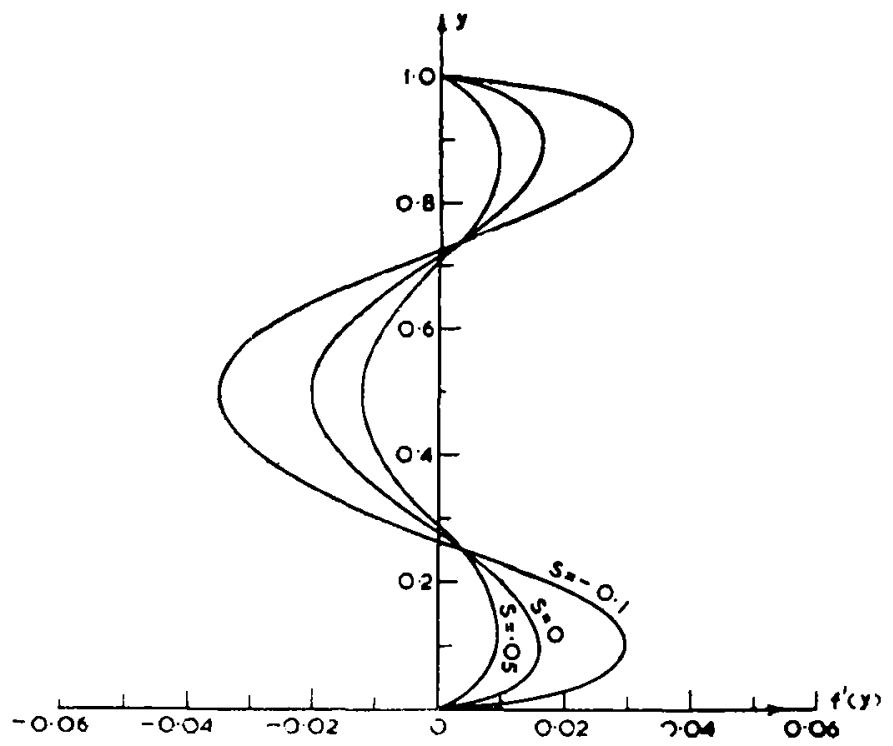

FKG. 4. Two discs oscillating: Steady radial velccity $f(6) S=-0 \cdot 1.0,-0 \cdot 65$ 
for $S=-0.1,0$ and +0.05 . For $S=-0.1$ it is zero on the planes $y=0.26$ and $y=0.72$. There is a radial outflow between these and the walls due to the predominance of centrifugal action. For $0.26<y<0.72$ there is an inflow as the pressure gradient is then the main factor. For $\mathrm{S}=+0.05$ it is zero on the plane $y=0.29$ and $y=0.71$.

The shearing stresses acting on the discs $z=0$ and $z=d$ are equal in magnitude but opposite in sign.

For small Reynolds numbers we have the shearing stresses,

$$
\begin{aligned}
& \mathrm{T}_{r z}=\mu \frac{r}{d} \frac{\Omega^{2}}{n} \frac{\mathrm{R}}{120}[4+\mathrm{R}(12 \mathrm{~S}-1) \sin 2 n t]+0\left(\mathrm{R}^{3}\right), \\
& \mathrm{T}_{\theta z}=-2 \mu \frac{r}{d} \Omega\left[\left(1+\frac{\mathrm{R}^{2}}{720}\right) \cos n t-\frac{\mathrm{R}}{12} \sin n t\right]+0\left(\mathrm{R}^{3}\right),
\end{aligned}
$$

and

$$
\begin{aligned}
\mathrm{T}_{r \theta}=- & \mu_{c} \frac{r^{2}}{\bar{d}^{2}} \frac{\Omega^{3}}{n} \frac{\mathrm{R}}{120}\left[8 \cos n t \div \mathrm{R}\left(12 \mathrm{~S}-\frac{5}{3} \sin n t\right.\right. \\
& +\mathrm{R}(12 \mathrm{~S}-1) \sin 3 n t]+0\left(\mathrm{R}^{3}\right) .
\end{aligned}
$$

\section{SUMMARY}

The flow of an incompressible non-Newtonian viscous tluid contained between two torsionally oscillating infinite parallel discs is investigated. The two specific cases studied are ( $i)$ one disc only oscillates wibile thr other is at rest and (ii) both discs oscillate with the same frequency and amplitude but in opposite directions. Assuming that the amplitude of oscillation, $\Omega / n$, is small and neglecting the squares and higher powers of $\Omega / n$, the equations of motion have been solved exactly for velocity and pressure satisfying all the boundary conditions. The effect of both positive and negative coefficients of cross-viscosity on the steady components of the flow has been represented graphically.

\section{ACKNOWLEDGEMENT}

The author is highly indebted to Prof. P. L. Bhatnagar for his constant guidance and kind help throughout the preparation of this paper.

\section{REFERENCE}

Rosenblat, S.

Journ. of Fluid Mech., 1960, 8, 388. 